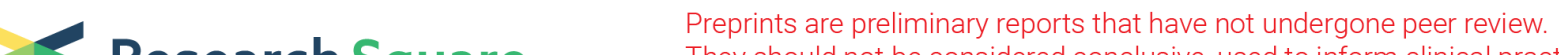 Research Square They should not be considered conclusive, used to inform clinical practice, or referenced by the media as validated information.
}

\section{Displacement-mediated bound states in the continuum in all-dielectric superlattice metasurfaces}

\section{Tan Shi}

Jinan University

\section{Zilan Deng ( $\nabla$ zilandeng@jnu.edu.cn )}

Institute of Photonics Technology, Jinan University https://orcid.org/0000-0003-3861-6014

Qing-An Tu

Jinan University

Yaoyu Cao

Jinan University

Xiangping Li

Jinan University

\section{Research}

Keywords: bound states in the continuum, all-dielectric metasurfaces, superlattice, Fano resonance

Posted Date: January 6th, 2021

DOl: https://doi.org/10.21203/rs.3.rs-139839/v1

License: (c) (1) This work is licensed under a Creative Commons Attribution 4.0 International License. Read Full License

Version of Record: A version of this preprint was published at PhotoniX on April 25th, 2021. See the published version at https://doi.org/10.1186/s43074-021-00029-x. 


\section{Abstract}

Bound states in the continuum (BICs) are localized states coexisting with extended waves inside the continuous spectrum range, which have infinite lifetimes without any radiation. To extract high-Q quasiBIC resonances from the symmetry-protected BIC for practical applications, symmetry-breaking approaches are usually exploited, either by slightly breaking the excitation field symmetry or structure symmetry. Here, we introduce an all-dielectric superlattice metasurface that can symmetry-compatibly convert BIC states into high-Q quasi-BIC modes based on the guided-mode resonance coupling by relative displacement tuning. The metasurface is composed of a superlattice of multiple nanobeams, supporting both magnetic mode and toroidal mode with large tunability. Both modes can interact with the incident continuum by mediating the displacement between nanobeams, which empowers dual asymmetric Fano resonances with high Q-factors. The bandwidth of the toroidal mode under TE-polarized incidences and that of the magnetic mode under TM-polarized incidences can be readily tuned by the local displacement between nanobeams in each unit cell. Such displacement-mediated BIC resonance is promising for various applications such as bio-molecule sensing and low threshold lasing.

\section{Introduction}

Metasurfaces, which consist of periodic arrays of carefully designed nanostructures, provide a versatile platform to accurately control the properties of light, including phase, amplitude, polarization and frequency spectrum of light [1]. In recent years, metasurfaces based on dielectric materials with high refractive index have been used to develop numerous compact optical devices due to their low loss properties, including flat lenses [2-4], beam deflectors [5, 6], holograms [7, 8], etc. Searching for the ultrahigh Q-factor resonant structures with strong mode coupling has attracted much attention recently [911]. In this respect, bound states in the continuums (BICs) emerged as a novel method of light confinement with infinity Q-factor [12,13]. BICs are highly localized states embedded inside the continuous spectrum coexisting with radiative modes, which can carry energy away, without any radiations like discrete and spatially bounded states $[12,13]$. True BICs are unavailable and can only exist in a mathematical model. When the system is disturbed a little bit from the ideal situation, the BIC mode would couple to the extended waves and leak into reachable Fano resonance with finite high Q-factor, which is the so-called "quasi-BIC" [14-17], or supercavity mode [18-20].

There are basically two types of BICs [12-41], namely, the symmetry-protected BICs and the resonancecoupled BICs [28-30]. The symmetry-protected BICs originate from the forbidden excitation of certain modes governed by the in-plane symmetry of the structure $[13,14]$. Ultra-high Q-factor resonance modes can be obtained by slightly breaking the symmetry of the structure, convert the BIC to ultra-sharp Fano resonances. The Fano resonances are ascribed to the asymmetric mode supported by the asymmetric structure, of which the electromagnetic field distribution slightly deviates from that of symmetric modes. And the Q-factor can be readily tuned by the degree of asymmetry [14]. Based on the symmetry-protected $\mathrm{BIC}$, most of the previous work converts the BIC into high-Q resonances by either slightly breaking the excitation field symmetry through oblique incidences $[31,32]$ or introducing structural asymmetry [14, 
21-27]. On the contrary, the resonance-coupled BICs originate from the linewidth vanishing by destructive interference of anti-crossing coupled resonance modes [15, 28, 29], which does not rely on the symmetry operations. However, most present resonance-coupled BICs are based on the engineering of multiple resonance modes supported by the unit-cell structure, which has multiple geometry degree of freedom and needs tedious researching procedure of proper parameters.

Here, we report a displacement-mediated superlattice metasurface that supports symmetry-compatible quasi-BICs based on the resonance coupling between the backward/forward guided modes through lattice transformation. The metasurface is composed of multiple parallel nanobeams with tailored global and local displacements, which are defined by the distance between nanobeams in adjacent unit cells and the distance between nanobeams within each unit cell, respectively (Fig. 1a). It can interplay with the incident continuum by transferring the simple lattice to a superlattice through relative displacement engineering of individual nanobeams. High Q-factor quasi-BIC resonance can be extracted from the ideal BIC by symmetry-compatibly making a slight difference between the local and global displacements. The bandwidth of the toroidal mode for transverse electric (TE)-polarized incidences and the magnetic mode for transverse magnetic (TM)-polarized incidences can be readily tuned by the local displacement between nanobeams in each unit cell. The resonance peak position of the toroidal quasi-BIC is linearly dependent on the local displacement, while that of the magnetic quasi-BIC remains unchanged with the local displacement, due to different mode characteristics. We envision that the displacement-mediated superlattice metasurfaces with BICs may facilitate many applications including high figure of merit biomolecule sensing and low threshold lasing.

\section{Results And Discussion}

Figure 1a shows the unit cell of the considered all-dielectric superlattice metasurface. It is composed of three identical nanobeams made of silicon $(n=3.47)$ with a length of $700 \mathrm{~nm}$, width of $150 \mathrm{~nm}$ and height of $110 \mathrm{~nm}$. The periods along the $x$ - and $y$-direction are $p_{x}=700 \mathrm{~nm}, p_{y}=750 \mathrm{~nm}$, respectively. The displacement between adjacent nanobeams within each unit cell is defined as the local displacement $\left(d_{1}\right)$, while the displacement between nanobeams in adjacent unit cells is defined as the global displacement $\left(\mathrm{d}_{2}\right)$. Here, we demonstrate the manipulation of quasi-BIC by introducing a displacement difference parameter $\left(\triangle d=d_{1}-d_{2}\right)$ to open a leaky channel of the BIC, which originates from the band folding of guided mode to the continuum. Figure $1 \mathrm{~b}$ shows the transmission spectra with respect to different $\triangle \mathrm{d}$ for the metasurface embedded in free space for TE-polarized normal incidences. For the $\triangle \mathrm{d}$ $=0$ (denoted as the red circle) case, the metasurface supports a BIC state with infinitely high Q-factors. The transmission spectrum manifests a smooth profile without a sharp dip due to the uncoupling between the external environment and the ideal BIC state. When $\triangle d \neq 0$ (denoted as green and blue circles), a narrow dip arises in the transmission spectra, which means the excitation of quasi-BIC state with a finite Q-factor. Noteworthily, another Fano resonance originated from magnetic dipole (MD) mode at shorter wavelengths does not vanish no matter how the spacing changes, and the bandwidth and the resonance peak position remain almost unchanged during the variation. The possible reason is that for 
TE-polarized incidences, the MD Fano resonance at shorter wavelengths is produced by the mutual coupling between adjacent nanobeams with each unit cell, which is only related to the local displacement, while the quasi-BIC resonance at the longer wavelength is caused not only by the coupling between nanobeams within each unit cell, but also the coupling between the three-nanobeam unit-cells, which are governed by both the local and global displacements, respectively. Similarly, the transmission spectra with variations of other structural parameters $\left(p_{x}, p_{y}, w_{x}, w_{y}\right.$ and $\left.h\right)$ are shown in Fig. $\mathrm{S} 1$ of Supplementary Information. As can be seen, those parameters only affect the peak position of double asymmetric Fano resonances, but cannot tune the linewidth to infinitesimal. Only by adjusting the relationship between local and global displacements $\left(\triangle d\right.$ or $\left.p_{x}\right)$ can we control the linewidth or even make it vanish. Figure $1 \mathrm{c}$ manifests the $\mathrm{Q}$-factor and the resonance wavelength of the quasi-BIC as functions of $\triangle \mathrm{d}$ around a BIC state. One can see that the linewidth of the quasi-BIC dwindles rapidly as $\triangle \mathrm{d}$ approaches zero, while the resonance peak shifts to the longer wavelength with the increasing of $\triangle \mathrm{d}$. The Q-factor of the BIC mode tends to infinity at $\triangle d=0$. We also calculated the band diagram and the Qfactor map of eigenmodes supported in the $\triangle d=0$ metasurface (See Fig. S2 in Supplementary Information for more details). As we can see, the BIC is originated from the resonance coupling between the forward and backward guided modes, reach their highest Q-factors at the $\Gamma$ point where they intersect with each other. To consider the practical conditions, results of the superlattice metasurface on a glass (n $=1.5$ ) substrate are shown in Fig. S3 of Supplementary Information. It can be seen that the Q-factor decreases with the existence of a substrate, since the substrate converts the BIC into a resonant state, leaking through the radiation channel in the substrate [38]. However, it remains high Q-factors and can be easily adjusted to the desired values by the displacement difference $\triangle \mathrm{d}$.

We recognize the BIC modes by utilizing multipole expansions [42-48] and near-field electromagnetic patterns of the modes. Figure $2 a$ shows the simulated transmittance and reflectance spectra of the superlattice metasurface with the displacement difference parameter $\triangle \mathrm{d}=-70 \mathrm{~nm}$, embedded in free space for TE-polarized normal incidences, where double asymmetric Fano resonances with high Q-factors can be observed at $\lambda=847 \mathrm{~nm}$ and $1148 \mathrm{~nm}$. Mode 1 refers to the MD Fano resonance at $847 \mathrm{~nm}$. Mode 2 refers to the quasi-BIC mediated by the parameter $\triangle \mathrm{d}$ at $1148 \mathrm{~nm}$. The spectral curves in Fig. 2a correspond to the specific case indicated by the dashed line of Fig. 1b. Figures $2 b$ and $2 c$ depict the extinction cross-section spectra by multipole expansions of the MD Fano resonance (resonance 1 ) and quasi-BIC resonance (resonance 2), respectively. We found that MD Fano resonance is indeed dominated by the MD mode, with smaller contributions from the electric quadrupole (EQ) mode. The quasi-BIC resonance is completely different, which is dominated by the toroidal (TD) mode, with smaller contributions from the magnetic quadrupole (MQ) mode, representing the radiation channel coupled to the external waves in our system. The locations of the strong scattering intensity of the multipoles are consistent with the spectra in Fig. 2a. By analyzing the near-field electromagnetic patterns of the modes, shown in Figs. $2 \mathrm{~d}$ and $2 \mathrm{e}$, we further confirm that the MD mode with antisymmetric distributions of polarization currents, accompanied by the coexistence of EQ mode with different strengths at $\lambda=847 \mathrm{~nm}$. Furthermore, the quasi-BIC resonance corresponds to TD mode with the dominant direction perpendicular to the incident light, accompanied by MQ mode with different strengths at $\lambda=1148 \mathrm{~nm}$. 
At the condition of $\triangle d \neq 0$, another symmetry-protected BIC is accompanied with our previous mentioned displacement-mediated quasi-BIC in the superlattice metasurface, which can be transferred to finitelinewdith quasi-BICs by varying the incident angle. The transmission spectra with respect to the angle and wavelength of TE-polarized incidence is depicted in Fig. 3a. The curve indicated by the green circle is the displacement-mediated quasi-BIC and the curve indicated by the blue circle is the symmetry-protected quasi-BIC mediated by the incident angle. Several appealing features can be observed from Fig. 3a. First, the linewidth and position of the green circle are determined by the parameter $\Delta \mathrm{d}$, which is also the largest linewidth of the quasi-BIC in the whole transmission spectra. If the parameter $\triangle d=0$, the quasiBIC will not emerge at any angles, which is completely confined. Second, for quasi-BICs (the blue circle) mediated by the incident angle, the line width of the quasi-BIC resonance absolutely vanishes because the system is completely symmetrical at normal incidences and does not couple with the external environment. We note that the maximum linewidth of the quasi-BIC produced by the incident angle is also determined by the parameter $\triangle \mathrm{d}$. If $\triangle \mathrm{d}=0$, the quasi-BIC mediated by the incident angle will not appear in the transmission spectrum. With the increase of $\triangle \mathrm{d}$, this quasi-BIC (the blue circle) appears in the case of oblique incidences, and the maximum linewidth of this quasi-BIC in the transmission spectrum increases. In other words, the quasi-BIC mediated by incident angles has the same resonance wavelength of the quasi-BIC caused by the displacement, with large angular dispersion. Moreover, the maximum linewidths of two types of the quasi-BICs appear at different positions in the transmission spectra, but their maximum linewidths are nearly identical and determined by the parameter $\triangle \mathrm{d}$. The band diagram of the TE mode is displayed in Fig. 3b, revealing large angular dispersions of the two types of quasi-BICs modes. In this regard, the band structure for the quasi-BIC case as shown in Fig. 3b reveals modes in the radiative region with high dispersion similar to that for the true BIC with a zero displacement in Fig. S2. Both are highly dispersive, but they have different diffraction lines since the lattice constant differs. Also, this is consistent with that if $\triangle d=0$, the quasi-BIC will not emerge at any angles. Figures $3 c$ and $3 d$ show their electric near-field patterns at the incident angle of $2^{\circ}$. Although the positions of the two quasi-BICs are very close to each other, the electromagnetic modes of the two quasi-BICs are different, which can be accurately analyzed by multipole expansions. Therefore, the extinction cross-section spectra by multipole expansions of the two quasi-BICs at the angle of $2^{\circ}$ are presented in Fig. S4 of Supplementary Information, revealing that the absorbing phenomenon can be largely attributed to the coexistence of dominant multipoles. The displacement-mediated quasi-BIC is dominated by the TD mode, coupled with concomitance of other multipoles including MD and MQ modes with distinct strengths, while the anglemediated quasi-BIC is produced by the concomitance of predominant multipoles including $\mathrm{MD}$ and EQ modes with unequal strengths. Figures $3 \mathrm{e}-3 \mathrm{~g}$ show the transmission spectra at angles of $2^{\circ}, 15^{\circ}$ and $30^{\circ}$, respectively, demonstrating that the two quasi-BICs mediated by the incident angle shift in the opposite directions with the increase of incident angles, which are promising to develop multiple angular dispersion applications.

Apart from the TE-polarized incidence, now we show that the displacement-mediated quasi-BIC is also applicable for the TM-polarized incidence (Fig. 4). Figure 4a shows the transmission and reflection spectra with parameter $\Delta \mathrm{d}=-70 \mathrm{~nm}$, revealing that one of the double asymmetric Fano resonances can 
be completely confined and converted into BICs with infinite Q-factors (Fig. 4b). Resonance 3 refers to the quasi-BIC mediated by the parameter $\triangle \mathrm{d}$ at $785 \mathrm{~nm}$. Resonance 4 refers to another TD Fano resonance at $955 \mathrm{~nm}$. The spectral curves in Fig. 4a correspond to the specified case indicated by the dashed line of Fig. 4b. The quasi-BIC near the wavelength of $785 \mathrm{~nm}$ can be obtained when the local displacement is tuned to be not equal to the global $\operatorname{displacement}(\triangle d \neq 0)$, that is, the simple lattice is transformed to a superlattice. When $\triangle d \neq 0$, a narrow dip arises in the transmission spectra, which means the excitation of quasi-BIC state, while the resonance position remains almost invariant. On the other hand, the spectra width of the TD Fano resonance near the wavelength of $955 \mathrm{~nm}$ is nearly steady with the change of $\triangle \mathrm{d}$. While the resonance position locates at the shortest wavelength at $\triangle d=0$, and shifts to longer wavelengths with the increasing of $|\triangle \mathrm{d}|$. More interestingly, we calculated extinction cross-section spectra by multipolar expansions for TM-polarized normal incidences as shown in Figs. 4c and 4d. It reveals that the dominant modes of the shorter-wavelength resonance modes and long-wavelength resonance modes are identical for TM and TE polarizations, but the intensity distribution is slightly different, with different directions of the mode due to the different polarizations. Figure 4c shows that the quasi-BIC near $785 \mathrm{~nm}$ mediated by the parameter $\triangle \mathrm{d}$ is dominated by the MD mode, coupled with smaller contributions from the EQ mode, which is similar to the modes of MD Fano resonance caused by the TE polarization in Fig. $2 \mathrm{~b}$. Then through the analysis of the electromagnetic field distribution in Fig. 4e, it is found that the mode is along the $y$-direction for the TM-polarized incidence, while the mode in Fig. $2 \mathrm{~d}$ is along the $x$-direction for the TE polarization. Different polarizations lead to different electromagnetic directions with identical modes. In addition, for the TM polarization (Fig. 4d), the Fano resonance near $955 \mathrm{~nm}$ is dominated by the TD mode, with smaller contributions from the MQ mode. Similarly, it can be seen from Figs. $4 \mathrm{f}$ and $2 \mathrm{e}$, the electromagnetic modes of the two are almost identical, but the directions are perpendicular to each other in the $x y$ plane. The variation of $\Delta \mathrm{d}$ can change the resonance position of the TD mode, but cannot change the resonance position of the MD mode. To summarize, for TM-polarized incidences, the resonance linewidth of the quasi-BIC dominated by the MD mode changes with the parameter $\triangle \mathrm{d}$, but the resonance position is basically unchanged, while the TD Fano resonance does not change the linewidth, but changes the position with the parameter $\triangle \mathrm{d}$. The quasi-BIC dominated by the MD mode under TM-polarized incidences can not only be controlled by $\triangle \mathrm{d}$, but also by other parameters $\left(p_{x}, p_{y}, w_{x}, w_{y}, h, \theta_{0}\right)$, as shown in Fig. $S 5$ of Supplementary Information, which demonstrates large tunability of the TM-polarized BIC modes.

\section{Conclusions}

In summary, we have proposed an all-dielectric superlattice metasurface composed of multiple parallel silicon nanobeams that can extract high Q-factor quasi-BIC resonances by transferring the simple lattice to a superlattice through displacement tuning between nanobeams. The displacement-mediated BIC modes are originated from the resonance coupling between anti-crossing guided modes of the metasurface and thus are symmetry compatible. When the local displacement is tuned to be slightly different from the global displacement, the superlattice metasurface supports both the TD quasi-BIC for the TE polarization and the MD quasi-BIC for the TM polarization with extremely high Q-factors. In our 
system, the resonance caused by MD mode does not shift in spectrum with the change of the displacement difference parameter $\triangle \mathrm{d}$, while the resonance caused by TD mode significantly shift with the change of $\triangle \mathrm{d}$. Moreover, a quasi-BIC with the maximum linewidth determined by the $\triangle \mathrm{d}$ emerges by changing the incident angle. Due to the high Q-factor of the quasi-BIC with its accessibility and controllability, our results provide a unique opportunity for facilitating many applications requiring extremely high Q-factors including ultra-high figure of merit sensing performance, nonlinear photonic metasurface and high-performance lasing.

\section{Abbreviations}

BICs: Bound states in the continuum; TE: Transverse electric; TM: Transverse magnetic; ED: Electric dipole; MD: Magnetic dipole; TD: Toroidal dipole; EQ: Electric quadrupole; MQ: Magnetic quadrupole

\section{Declarations}

\section{Acknowledgements}

We thank Yi Xu and Shuai Wang for useful discussions.

\section{Author contributions}

All the authors have accepted responsibility for the entire content of this submitted manuscript and approved submission.

\section{Funding}

The authors acknowledge the funding support provided by the National Key R\&D Program of China (2018YFB1107200), Guangdong Basic and Applied Basic Research Foundation (2020A1515010615), the Fundamental Research Funds for the Central Universities (21620415), the National Natural Science Foundation of China (NSFC) $(62075084,61522504,61420106014,11734012$, and 11574218), the Guangdong Provincial Innovation and Entrepreneurship Project (2016ZT06D081).

\section{Availability of data and materials}

The datasets and figures used and analyzed during the current study are available from the corresponding author on reasonable request.

\section{Competing interests}

The authors declare no competing financial interests.

\section{Author details}


${ }^{1}$ Guangdong Provincial Key Laboratory of Optical Fiber Sensing and Communications, Institute of Photonics Technology, Jinan University, Guangzhou, 510632, China.

\section{References}

1. Yu NF, Genevet P, Kats MA, Aieta F, Tetienne JP, Capasso F, et al. Light propagation with phase discontinuities: generalized laws of reflection and refraction. Science. 2011;334(6054):333-7.

2. Khorasaninejad M, Chen WT, Devlin RC, Oh J, Zhu AY, Capasso F. Metalenses at visible wavelengths: Diffraction-limited focusing and subwavelength resolution imaging. Science. 2016;352(6290):11904.

3. Wang S, Wu PC, Su VC, Lai YC, Chen MK, Kuo HY, et al. A broadband achromatic metalens in the visible. Nat Nanotechnol. 2018;13(3):227-32.

4. Zou X, Zheng G, Yuan Q, Zang W, Chen R, Li T, et al. Imaging based on metalenses. PhotoniX. 2020;1(1).

5. Sell D, Yang J, Doshay S, Yang R, Fan JA. Large-angle, multifunctional metagratings based on freeform multimode geometries. Nano Lett. 2017;17(6):3752-7.

6. Shi T, Wang Y, Deng ZL, Ye X, Dai Z, Cao Y, et al. All-Dielectric Kissing-Dimer Metagratings for Asymmetric High Diffraction. Adv Opt Mater. 2019;7(24).

7. Zheng G, Muhlenbernd H, Kenney M, Li G, Zentgraf T, Zhang S. Metasurface holograms reaching $80 \%$ efficiency. Nat Nanotechnol. 2015;10(4):308-12.

8. Deng ZL, Deng J, Zhuang X, Wang S, Shi T, Wang GP, et al. Facile metagrating holograms with broadband and extreme angle tolerance. Light: Sci Appl. 2018;7(1):78.

9. Yang Y, Kravchenko II, Briggs DP, Valentine J. All-dielectric metasurface analogue of electromagnetically induced transparency. Nat Commun. 2014;5:5753.

10. Fernandez-Bravo A, Wang D, Barnard ES, Teitelboim A, Tajon C, Guan J, et al. Ultralow-threshold, continuous-wave upconverting lasing from subwavelength plasmons. Nat Mater. 2019;18(11):11726.

11. Zhao R, Huang L, Wang Y. Recent advances in multi-dimensional metasurfaces holographic technologies. PhotoniX. 2020;1(1).

12. Stillinger FH, Herrick DR. Bound states in the continuum. Phys Rev A. 1975;11(2):446-54.

13. Hsu CW, Zhen B, Stone AD, Joannopoulos JD, Soljačić M. Bound states in the continuum. Nat Rev Mater. 2016;1(9).

14. Koshelev K, Lepeshov S, Liu M, Bogdanov A, Kivshar Y. Asymmetric Metasurfaces with High-Q Resonances Governed by Bound States in the Continuum. Phys Rev Lett. 2018;121(19):193903.

15. Azzam SI, Shalaev VM, Boltasseva A, Kildishev AV. Formation of Bound States in the Continuum in Hybrid Plasmonic-Photonic Systems. Phys Rev Lett. 2018;121(25):253901.

16. Liu M, Choi DY. Extreme Huygens' Metasurfaces Based on Quasi-Bound States in the Continuum. Nano Lett. 2018;18(12):8062-9. 
17. Bogdanov AA, Koshelev KL, Kapitanova PV, Rybin MV, Gladyshev SA, Sadrieva ZF, et al. Bound states in the continuum and Fano resonances in the strong mode coupling regime. Advanced Photonics. 2019;1(01).

18. Rybin MV, Koshelev KL, Sadrieva ZF, Samusev KB, Bogdanov AA, Limonov MF, et al. High-Q Supercavity Modes in Subwavelength Dielectric Resonators. Phys Rev Lett. 2017;119(24):243901.

19. Carletti L, Koshelev K, De Angelis C, Kivshar Y. Giant Nonlinear Response at the Nanoscale Driven by Bound States in the Continuum. Phys Rev Lett. 2018;121(3):033903.

20. Koshelev K, Kruk S, Melik-Gaykazyan E, Choi JH, Bogdanov A, Park HG, et al. Subwavelength dielectric resonators for nonlinear nanophotonics. Science. 2020;367(6475):288-92.

21. Liu Z, Xu Y, Lin Y, Xiang J, Feng T, Cao Q, et al. High-Q Quasibound States in the Continuum for Nonlinear Metasurfaces. Phys Rev Lett. 2019;123(25).

22. Koshelev K, Tang Y, Li K, Choi D-Y, Li G, Kivshar Y. Nonlinear Metasurfaces Governed by Bound States in the Continuum. ACS Photonics. 2019;6(7):1639-44.

23. Xu L, Zangeneh Kamali K, Huang L, Rahmani M, Smirnov A, Camacho-Morales R, et al. Dynamic Nonlinear Image Tuning through Magnetic Dipole Quasi-BIC Ultrathin Resonators. Adv Sci. 2019;6(15):1802119.

24. Leitis A, Tittl A, Liu M, Lee BH, Gu MB, Kivshar YS, et al. Angle-multiplexed all-dielectric metasurfaces for broadband molecular fingerprint retrieval. Sci Adv. 2019;5(5):eaaw2871.

25. Tittl A, Leitis A, Liu M, Yesilkoy F, Choi DY, Neshev DN, et al. Imaging-based molecular barcoding with pixelated dielectric metasurfaces. Science. 2018;360(6393):1105-9.

26. Yesilkoy F, Arvelo ER, Jahani Y, Liu M, Tittl A, Cevher V, et al. Ultrasensitive hyperspectral imaging and biodetection enabled by dielectric metasurfaces. Nat Photonics. 2019;13(6):390-6.

27. Kupriianov AS, Xu Y, Sayanskiy A, Dmitriev V, Kivshar YS, Tuz VR. Metasurface Engineering through Bound States in the Continuum. Phys Rev Appl. 2019;12(1).

28. Kodigala A, Lepetit T, Gu Q, Bahari B, Fainman Y, Kante B. Lasing action from photonic bound states in continuum. Nature. 2017;541(7636):196-9.

29. Hsu CW, Zhen B, Lee J, Chua SL, Johnson SG, Joannopoulos JD, et al. Observation of trapped light within the radiation continuum. Nature. 2013;499(7457):188-91.

30. Linton CM, Mclver P. Embedded trapped modes in water waves and acoustics. Wave Motion. 2007;45(1-2):16-29.

31. Fan K, Shadrivov IV, Padilla WJ. Dynamic bound states in the continuum. Optica. 2019;6(2).

32. Ha ST, Fu YH, Emani NK, Pan Z, Bakker RM, Paniagua-Dominguez R, et al. Directional lasing in resonant semiconductor nanoantenna arrays. Nat Nanotechnol. 2018;13(11):1042-7.

33. Doeleman HM, Monticone F, den Hollander W, Alù A, Koenderink AF. Experimental observation of a polarization vortex at an optical bound state in the continuum. Nat Photonics. 2018;12(7):397-401.

34. Sadrieva Z, Frizyuk K, Petrov M, Kivshar Y, Bogdanov A. Multipolar origin of bound states in the continuum. Phys Rev B. 2019;100(11). 
35. Jin J, Yin X, Ni L, Soljacic M, Zhen B, Peng C. Topologically enabled ultrahigh-Q guided resonances robust to out-of-plane scattering. Nature. 2019;574(7779):501-4.

36. Liu W, Wang B, Zhang Y, Wang J, Zhao M, Guan F, et al. Circularly Polarized States Spawning from Bound States in the Continuum. Phys Rev Lett. 2019;123(11):116104.

37. Marinica DC, Borisov AG, Shabanov SV. Bound States in the continuum in photonics. Phys Rev Lett. 2008;100(18):183902.

38. Sadrieva ZF, Sinev IS, Koshelev KL, Samusev A, lorsh IV, Takayama O, et al. Transition from Optical Bound States in the Continuum to Leaky Resonances: Role of Substrate and Roughness. ACS Photonics. 2017;4(4):723-7.

39. Zhen B, Hsu CW, Lu L, Stone AD, Soljacic M. Topological nature of optical bound states in the continuum. Phys Rev Lett. 2014;113(25):257401.

40. Lee S-G, Kim S-H, Kee C-S. Bound states in the continuum (BIC) accompanied by avoided crossings in leaky-mode photonic lattices. Nanophotonics. 2020;9(14):4373-80.

41. Yin X, Jin J, Soljačić M, Peng C, Zhen B. Observation of unidirectional bound states in the continuum enabled by topological defects. arXiv preprint arXiv:190411464. 2019.

42. Evlyukhin AB, Reinhardt C, Chichkov BN. Multipole light scattering by nonspherical nanoparticles in the discrete dipole approximation. Phys Rev B. 2011;84(23):235429.

43. He Y, Guo G, Feng T, Xu Y, Miroshnichenko AE. Toroidal dipole bound states in the continuum. Phys Rev B. 2018;98(16):161112.

44. Liu W, Kivshar YS. Multipolar interference effects in nanophotonics. Philos Trans A Math Phys Eng Sci. 2017;375(2090):1-10.

45. Liu W, Kivshar YS. Generalized Kerker effects in nanophotonics and meta-optics. Opt Express. 2018;26(10):13085-105.

46. Nieto-Vesperinas M, Gomez-Medina R, Saenz JJ. Angle-suppressed scattering and optical forces on submicrometer dielectric particles. J Opt Soc Am A-Opt Image Sci Vis. 2011;28(1):54-60.

47. Radescu EE, Vaman G. Exact calculation of the angular momentum loss, recoil force, and radiation intensity for an arbitrary source in terms of electric, magnetic, and toroid multipoles. Phys Rev E Stat Nonlin Soft Matter Phys. 2002;65:046609.

48. Savinov V, Fedotov VA, Zheludev NI. Toroidal dipolar excitation and macroscopic electromagnetic properties of metamaterials. Phys Rev B. 2014;89(20):205112.

\section{Figures}


a

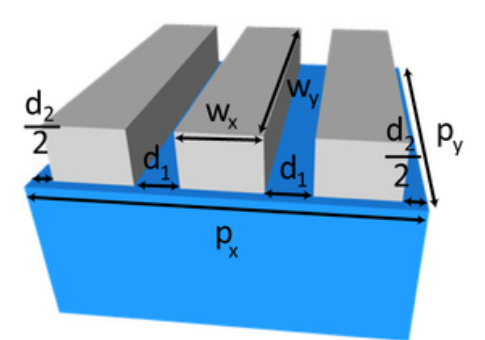

$\triangle d=-200 n m$

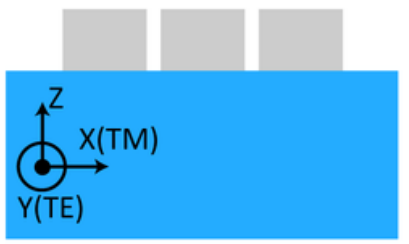

b

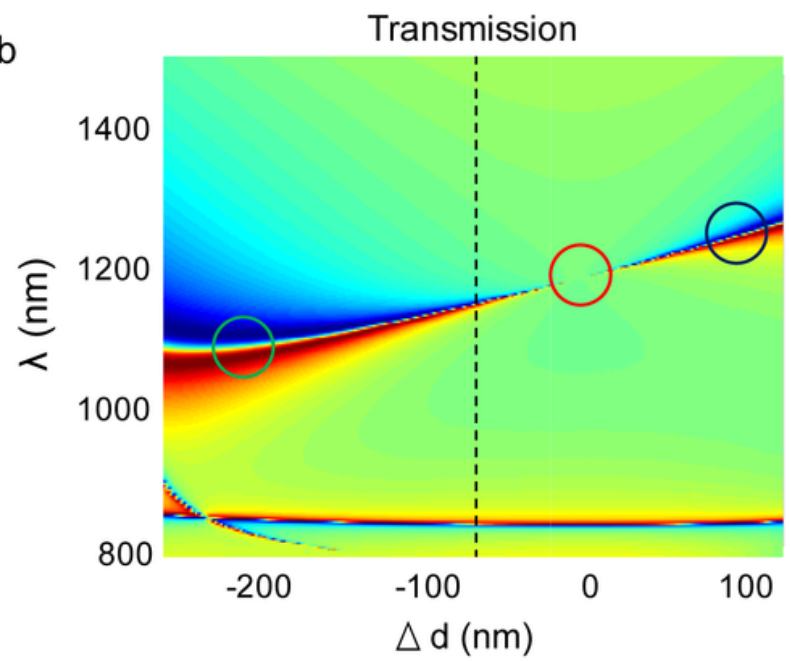

$\bigcirc \mathrm{d}=0 \mathrm{~nm}$
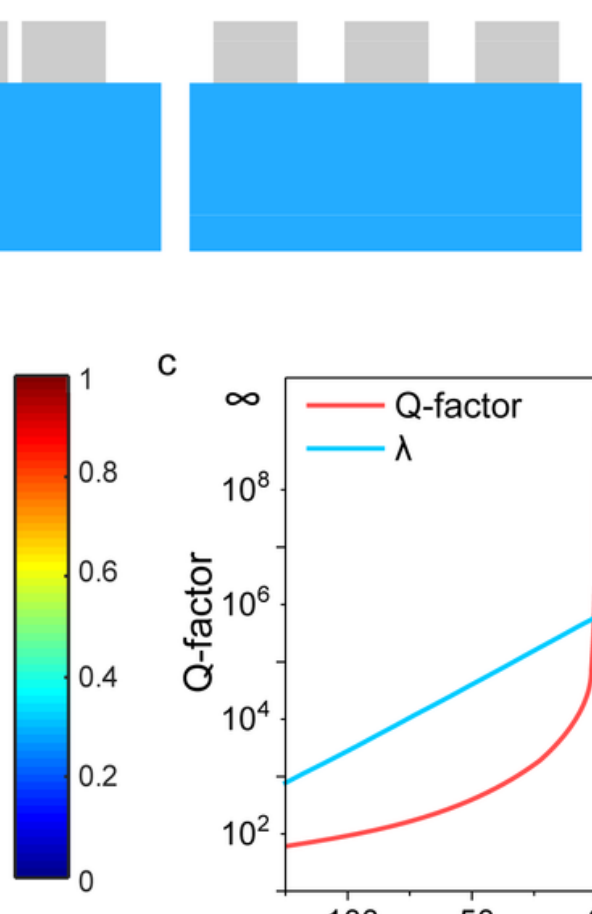

C

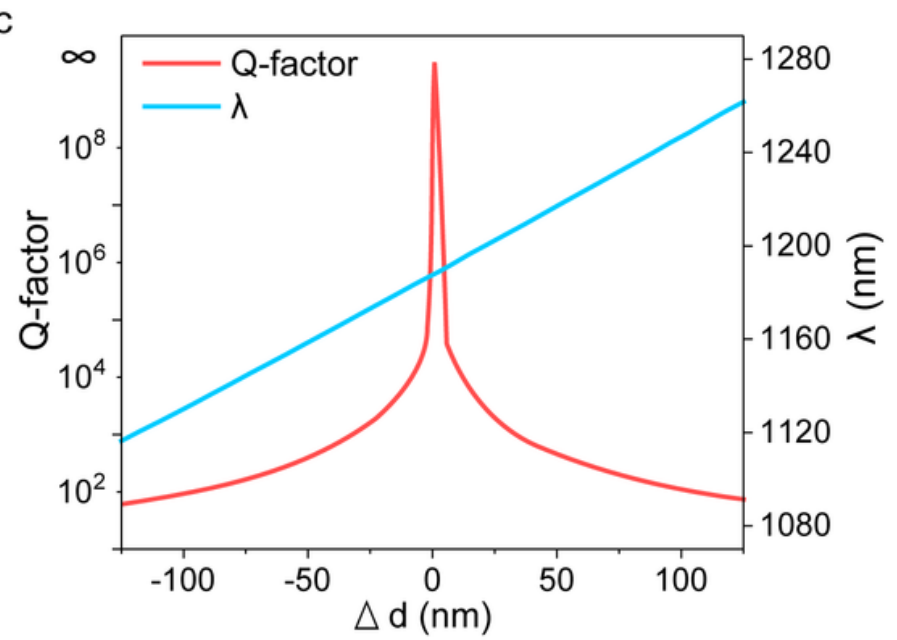

\section{Figure 1}

(a) Schematic of the all-dielectric superlattice metasurface supporting displacement-mediated quasiBICs. The unit cell consists of three silicon nanobeams with different local displacement (d1) and global displacement $(\mathrm{d} 2)$. Three representative unit cells with different $\triangle \mathrm{d}$ are shown on the right panel: $\triangle \mathrm{d}=$ $-200 \mathrm{~nm}$ (green circle), $\triangle \mathrm{d}=0 \mathrm{~nm}$ (red circle), and $\triangle \mathrm{d}=100 \mathrm{~nm}$ (blue circle). (b) The transmission spectra with respect to $\triangle \mathrm{d}$. The color circle symbols represent the resonance peak of quasi-BIC with three different $\triangle \mathrm{d}$ as indicated in (a). (c) Dependence of the Q-factor and resonance wavelength of the quasi$\mathrm{BIC}$ mode on $\triangle \mathrm{d}$. 
a
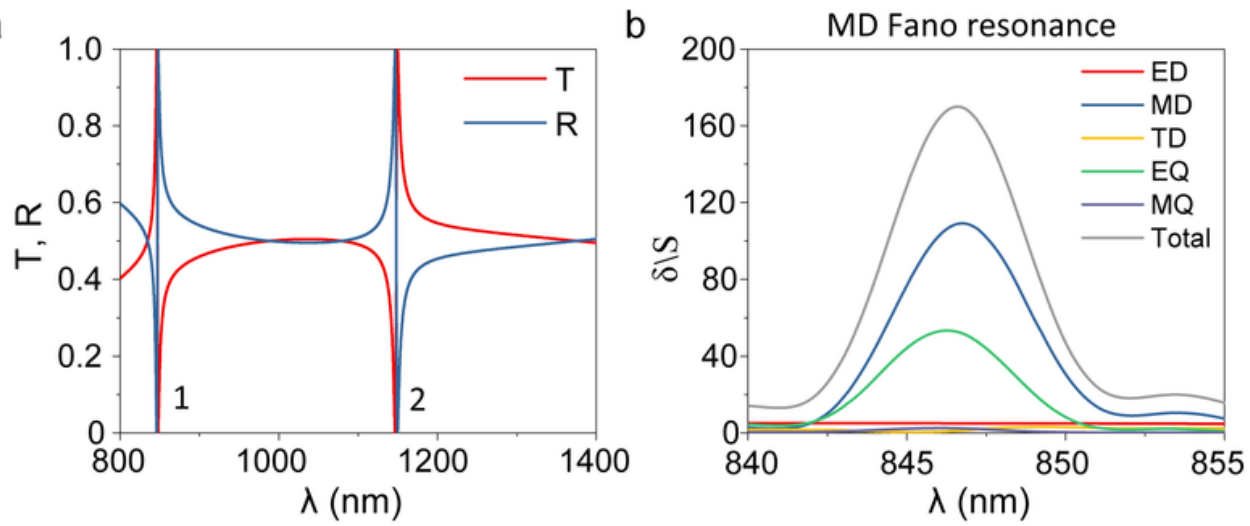

d

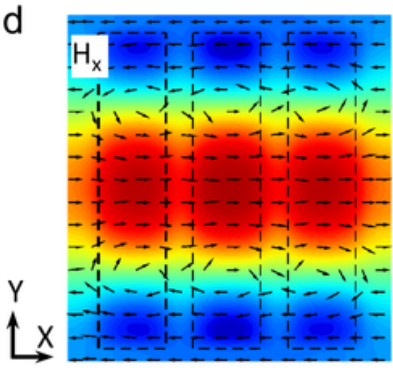

$\mathrm{e}$

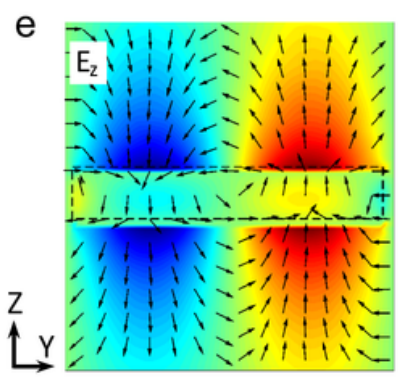

C
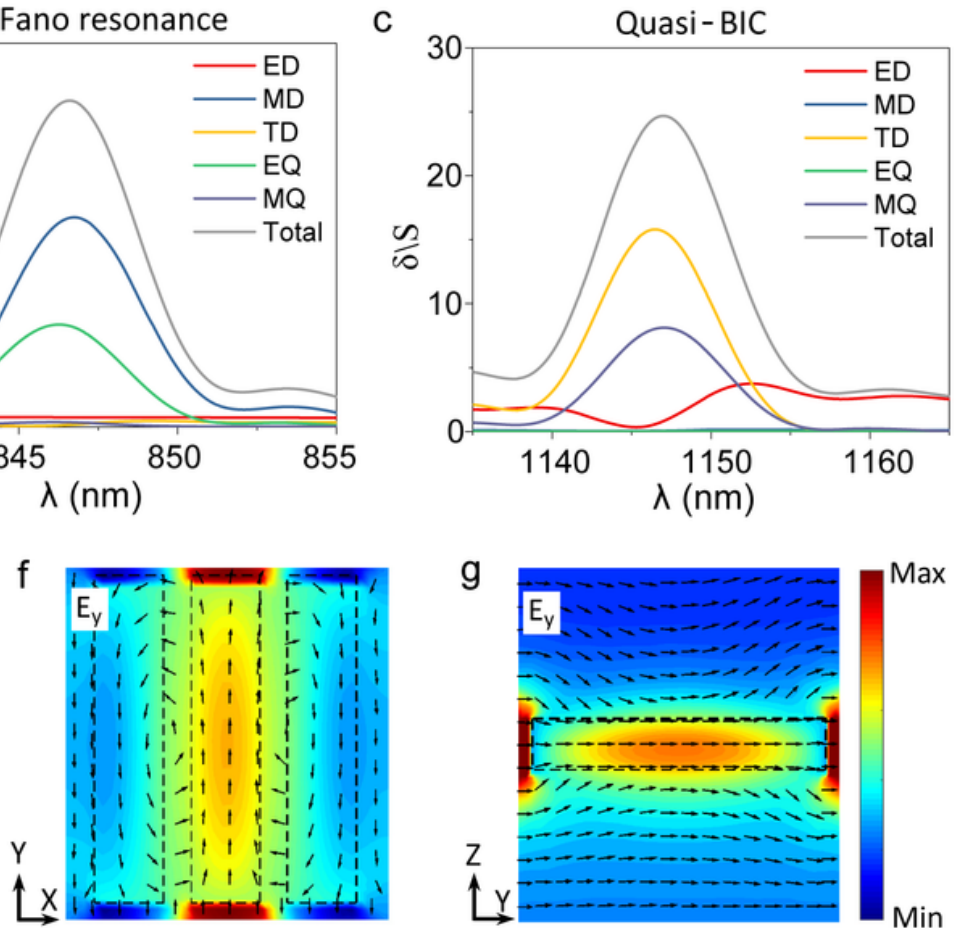

g

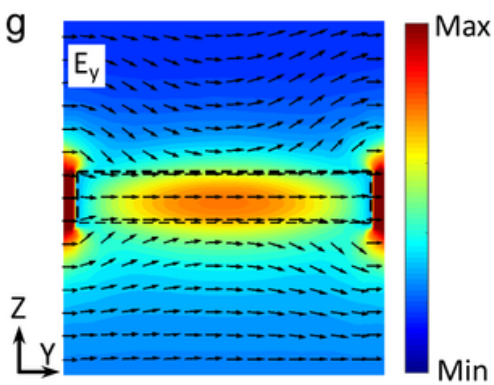

Figure 2

(a) Transmission (red curve) and reflection (blue curve) spectra of the superlattice metasurface under TEpolarized normal incidences. Parameters of the structure: $p x=700 \mathrm{~nm}, \mathrm{py}=750 \mathrm{~nm}, \mathrm{wx}=150 \mathrm{~nm}$, wy = $700 \mathrm{~nm}, \mathrm{~h}=110 \mathrm{~nm}, \mathrm{~d} 1=60 \mathrm{~nm}$, and d2 = $130 \mathrm{~nm}$. (b and c) Extinction cross-section spectra by multipolar expansion of the superlattice metasurface at the two resonant modes shown in (a). ED (red line) denotes electric dipole; MD (blue line) denotes magnetic dipole; TD (yellow line) denotes toroidal dipole; EQ (green line) denotes electric quadrupole; MQ (violet line) denotes magnetic quadrupole and the gray line denotes total scattering intensity. ( $d$ and e) The electric near-field distribution at $\lambda=847 \mathrm{~nm}$. $(f$ and g) The electric near-field distribution at $\lambda=1148 \mathrm{~nm}$. The xy plane is at $\mathrm{z}=55 \mathrm{~nm}$ (The middle of the nanobeams) above the base of the silicon nanobeams. The yz plane passes through the center of the unit cell. 

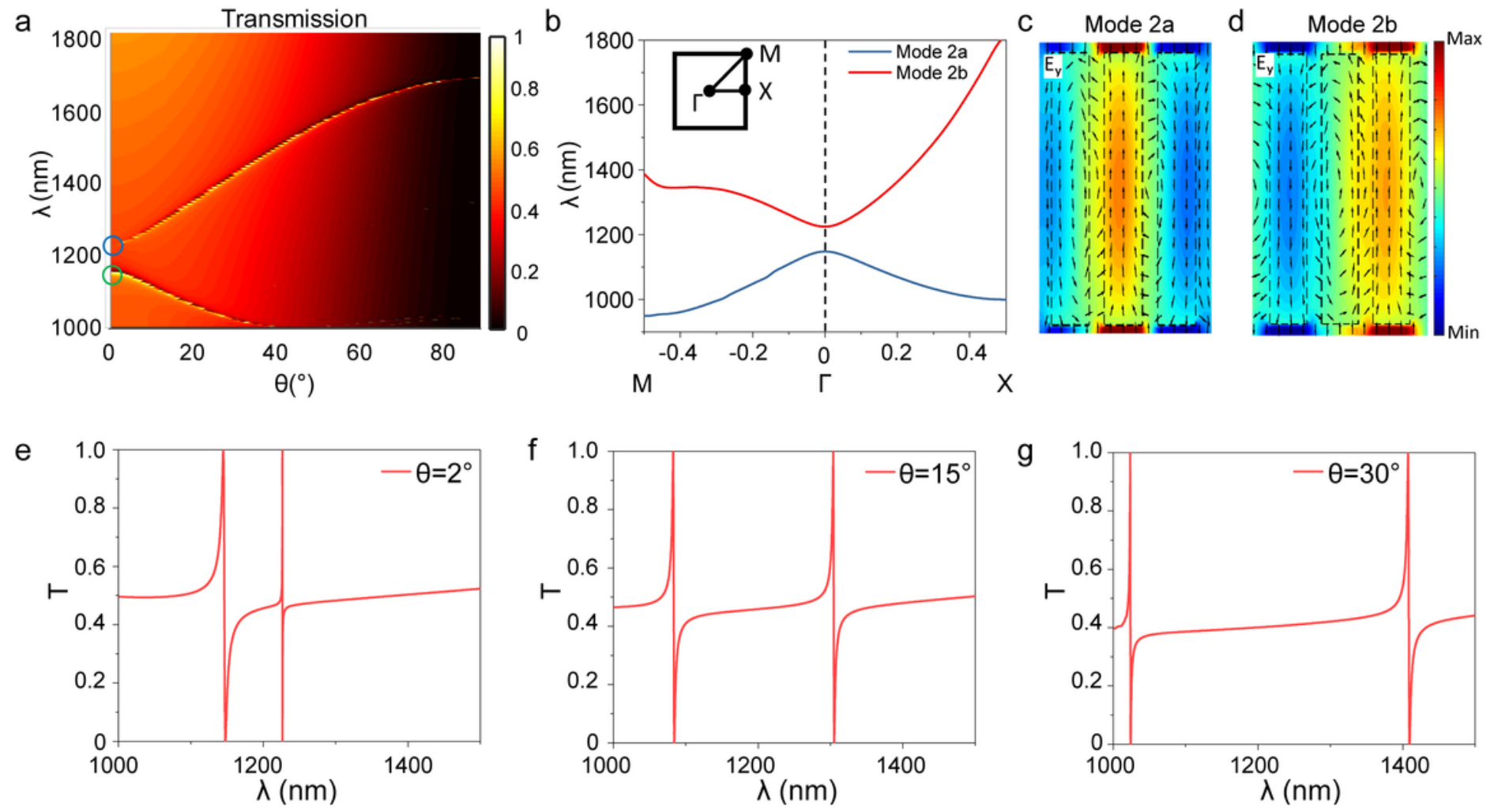

Figure 3

(a) Transmission spectra with respect to the angle and wavelength of incidence of the superlattice metasurface with the same parameters as Fig. 2a. (b) Band structure of the superlattice metasurface slab along $М \Gamma$ and $\Gamma X$ directions. (c and $d$ ) The electric near-field distribution at two different quasi-BICs resonances at the incident angle of $2^{\circ}$. (e, $f$ and $g$ ) The simulated transmission spectra for varying incident angles $2^{\circ}, 15^{\circ}$, and $30^{\circ}$, respectively.
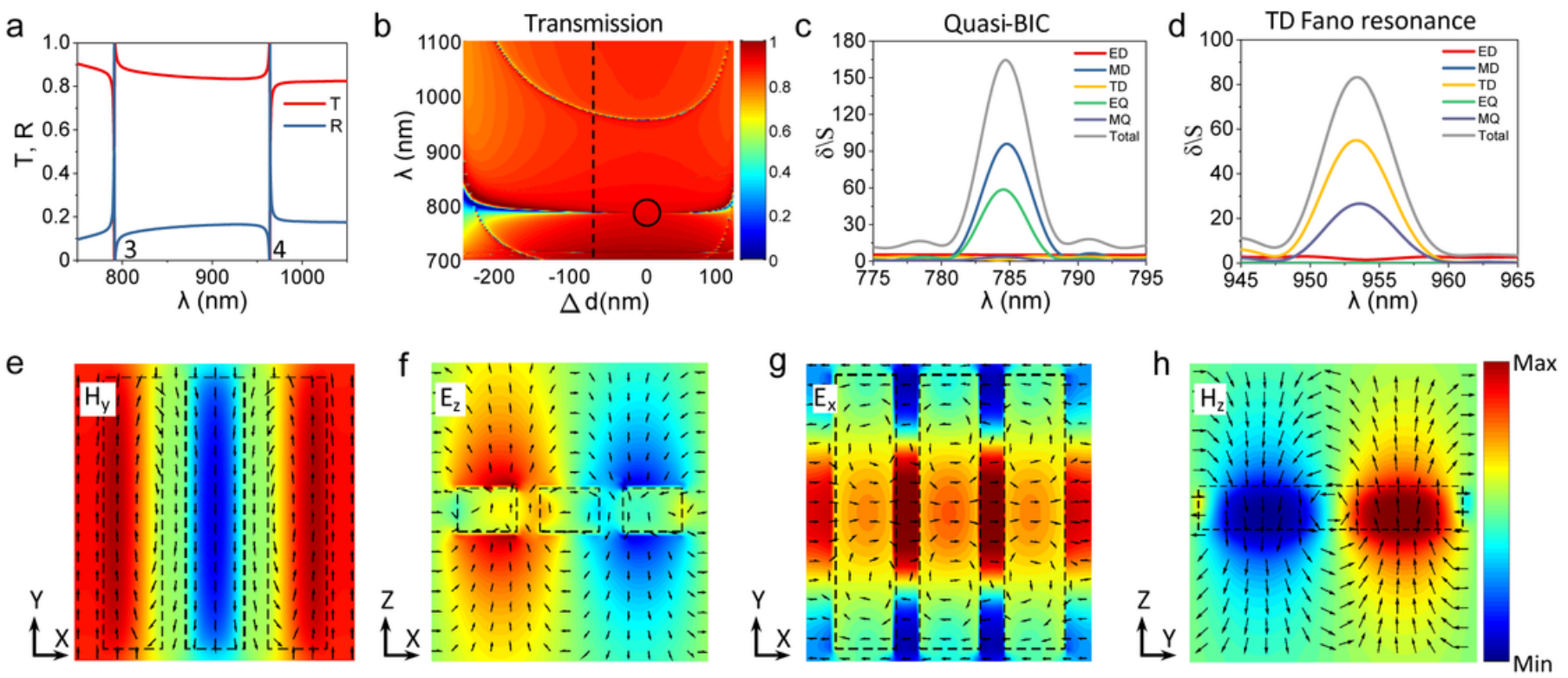


\section{Figure 4}

Quasi-BIC modes for TM-polarized normal incidences. (a) Transmission (red curve) and reflection (blue curve) spectra of the superlattice metasurface with the same parameters as Fig. 2a. (b) The transmission spectra with varying displacement $\triangle \mathrm{d}$. ( $\mathrm{c}$ and $\mathrm{d}$ ) Extinction cross-section spectra by multipolar expansion of the superlattice metasurface with different modes for TM-polarized normal incidences. (e and $f$ ) The near-field distribution of resonant mode 3 at $\lambda=785 \mathrm{~nm}$. ( $\mathrm{g}$ and $\mathrm{h}$ ) The near-field distribution of resonant mode 4 at $\lambda=955 \mathrm{~nm}$.

\section{Supplementary Files}

This is a list of supplementary files associated with this preprint. Click to download.

- BICPhotoniXSupplementaryInformation.docx 